\title{
Mining the bladder cancer-associated genes by an integrated strategy for the construction and analysis of differential co-expression networks
}

\author{
Su-Ping Deng, Lin Zhu, De-Shuang Huang* \\ From 10th International Symposium on Bioinformatics Research and Applications (ISBRA-14) \\ Zhangjiajie, China. 28-30 June 2014
}

\begin{abstract}
Background: Bladder cancer is the most common malignant tumor of the urinary system and it is a heterogeneous disease with both superficial and invasive growth. However, its aetiological agent is still unclear. And it is indispensable to find key genes or modules causing the bladder cancer. Based on gene expression microarray datasets, constructing differential co-expression networks (DCNs) is an important method to investigate diseases and there have been some relevant good tools such as R package 'WGCNA', 'DCGL'.

Results: Employing an integrated strategy, 36 up-regulated differentially expressed genes (DEGs) and 356 downregulated DEGs were selected and main functions of those DEGs are cellular physiological precess(24 up-regulated DEGs; 167 down-regulated DEGs) and cellular metabolism (19 up-regulated DEGs; 104 down-regulated DEGs). The up-regulated DEGs are mainly involved in the the pathways related to "metabolism". By comparing two DCNs between the normal and cancer states, we found some great changes in hub genes and topological structure, which suggest that the modules of two different DCNs change a lot. Especially, we screened some hub genes of a differential subnetwork between the normal and the cancer states and then do bioinformatics analysis for them.

Conclusions: Through constructing and analyzing two differential co-expression networks at different states using the screened DEGs, we found some hub genes associated with the bladder cancer. The results of the bioinformatics analysis for those hub genes will support the biological experiments and the further treatment of the bladder cancer.
\end{abstract}

\section{Background}

The morbidity of bladder cancer is in the first place among the cancers of urinary system. The bladder cancer cells can spread by breaking away from the original tumor. They can spread through the blood vessels to the liver, lungs and bones. However, its causes are not yet clear. The bladder cancer is a heterogeneous disease that shows both superficial and invasive growth [1,2]. Superficial tumors frequently recur and may progress to invasive growth. A part that warrants better treatment regimes bladder cancer is also a good model system to study tumor initiation and progression. To gain insights into

\footnotetext{
* Correspondence: dshuang@tongji.edu.cn

College of Electronics and Information Engineering, Tongji University, Caoan
} Road, Shanghai, China

the molecular biology of these processes, we performed gene expression analyses to get some important information about bladder cancer-associated genes.

Systems biology is an emerging approach applied to biomedical and biological scientific research. It is a biology-based inter-disciplinary field of study that focuses on complex interactions within biological systems, using a holistic approach to biological and biomedical research [3-5]. Network biology is a new way of representation and analysis of biological information processing, which understands life as a network. In fact, the network biology is a branch of the systems biology.

Differential co-expression network (DCN) is one of biological networks. A gene co-expression network has emerged as a novel holistic approach for microarray 
analysis [6-9]. Stuart et al. [6] and Bergmann et al. [7] separately constructed the gene co-expression network that connected genes whose expression profiles were similar across different organisms. A human network was analyzed by Leeet al. [8] with functional grouping and cluster analysis. van Noort et al. [9] demonstrated the small-world and scale-free architecture of the yeast co-expression network. They showed that functionally related genes are frequently co-expressed across organisms constituting conserved transcription modules.

We wanted to explore transcriptional changes in terms of gene interactions rather than at the level of individual genes. In the end, we constructed two gene co-expression networks and sought to find cancer-induced changes in the network. The identification of co-expressed pairs in tumor and normal tissues led to the construction of two distinct networks that represent tumor and normal states, respectively. We expected that biological changes would be reflected in transcriptional changes, which could be identified by comparing the two co-expression networks. In the transcriptome analysis, differential coexpression analysis (DCEA) is emerging as a unique complement to traditional differential expression analysis. DCEA investigates differences in gene interconnection by calculating the expression correlation changes of gene pairs between two conditions. The rationale behind differential co-expression analysis is that changes in gene co-expression patterns between two contrasting phenotypes (e.g., healthy and disease) provide hints regarding the disrupted regulatory relationships or affected regulatory subnetworks specific to the phenotype of interest (in this case, the disease phenotype). Therefore, among the many growing directions of DCEA, there is the so-called "differential regulation analysis"(DRA), which integrates the transcription factor (TF)-to-target information to probe upstream regulatory events that account for the observed co-expression changes. Recently, many researchers have integrated differential co-expression and differential expression concepts to propose a novel Regulatory Impact Factor (RIF) that can be used to prioritize disease-causative TFs $[10,11]$.

In addition, a lot of researchers have begun to perform differential co-expression analyses of microRNAs $[12,13]$. Currently, some tools have been developed for differential expression analysis based on microarray, such as $\mathrm{R}$ packages "LIMMA" [14], "SAMR" [15], "WGCNA" [16] and so on.

In our study, we collected microarray datasets of bladder cancer from GEO http://www.ncbi.nlm.nih.gov/geo/ to analyze the datasets by an integrated strategy including some functions of SAMR [15], WGCNA [16], Cytoscape [17] and other packages. We selected some DEGs at two different state (normal and cancer) and constructed two DCNs. Through the comparisons between two DCNs of two different states, we found some hub genes associated with the bladder cancer.

\section{Results}

The datasets of gene expression Affymetrix microarray of bladder cancer [GEO: GSE3167] were download from the GEO database of NCBI [18]. It has 18 samples, [GSM71019-GSM71027] are from the normal tissues and the other 9 samples are from the cancer tissues. The datasets were processed by an integrated strategy. Some DEGs were selected and constructed two DCNs. In the end, simple analysis was applied to the two DCNs and some hub genes were found through comparing two DCNs at different states.

\section{Normalization of the microarray datasets}

In order to get high-quality and strong-expression genes for the convenience of the following data processing, we normalized the microarray dataset using medians (Additional file 1 shows the comparison between before and after normalization). After normalization, the expression values are in the better order. We also discovered the distribution of the expression datasets (Figure 1).

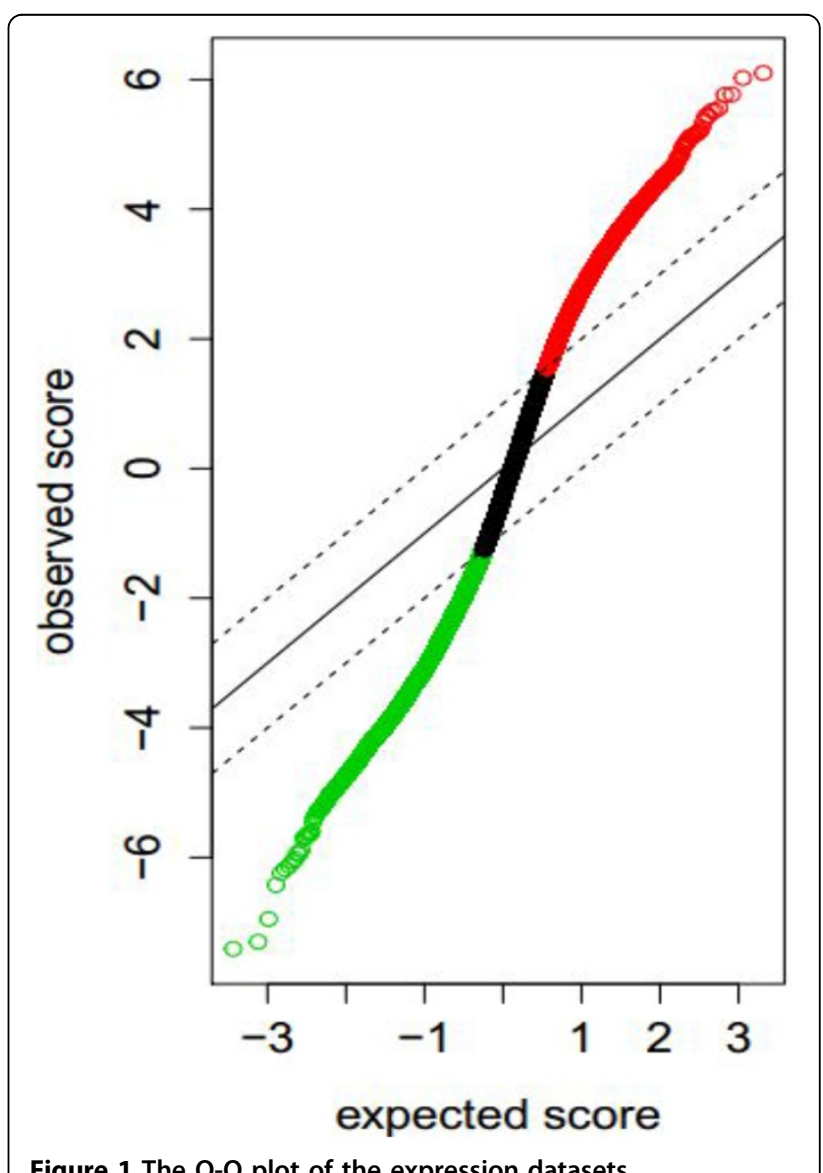




\section{Selection and bioinformatical analysis of DEGs}

After preprocessing the microarray datasets including the above normalization, some DEGs were selected using the R package "SAMR" (nperms(Number of permutations used to estimate false discovery rates) $=100$; del (Value of delta to define cutoff rule) $=2.5$ ). 36 upregulated genes and 356 down-regulated genes were picked out (See Additional file 2).
Next, we did bioinformatics analysis of those DEGs including GO function enrichment using a online tool AmiGO [19]. A part of GO enrichment results are showed in Figure 2. From the GO function enrichment results, we can easily find that the main functions of the up-regulated DEGs are nitrogen compound metabolic process (GO:0006807)(20 genes), heterocycle metabolic process (GO:0046483) (20 genes), cellular aromatic

\begin{tabular}{|c|c|c|c|c|c|}
\hline Term & $\begin{array}{l}\text { Background } \\
\text { frequency }\end{array}$ & $\begin{array}{l}\text { Sample } \\
\text { frequency }\end{array}$ & Expected & H- & P-value \\
\hline primary metabolic process (G0:0044238) & 8458 & 28 & $1.435 \mathrm{e}+01$ & + & $3.336 \mathrm{e}-04$ \\
\hline organic substance metabolic process (G0:0071704) & 8712 & 28 & $1.478 \mathrm{e}+01$ & + & $6.500 e^{-04}$ \\
\hline cellular metabolic process (G0:0044237) & 8327 & 27 & $1.413 e+01$ & + & $1.097 \mathrm{e}-03$ \\
\hline metabolic process (GO:0008152) & 9545 & 29 & $1.620 \mathrm{e}+01$ & + & $1.121 \mathrm{e}-03$ \\
\hline heterocycle metabolic process (G0:0046483) & 4765 & 20 & $8.086 \mathrm{e}+00$ & + & $1.123 e-03$ \\
\hline cellular aromatic compound metabolic process (G0:0006725) & 4768 & 20 & $8.091 \mathrm{e}+00$ & + & $1.134 \mathrm{e}-03$ \\
\hline cellular process (GO:0009987) & 13329 & 34 & $2.262 \mathrm{e}+01$ & + & $1.669 \mathrm{e}-03$ \\
\hline organic cyclic compound metabolic process (GO:1901360) & 4996 & 20 & $8.478 \mathrm{e}+00$ & + & $2.335 \mathrm{e}-03$ \\
\hline nucleobase-containing compound metabolic process (G0:0006139) & 4581 & 19 & $7.774 e+00$ & + & $2.603 \mathrm{e}-03$ \\
\hline cellular macromolecule metabolic process (G0:0044260) & 6145 & 22 & $1.043 e+01$ & + & $4.064 e-03$ \\
\hline macromolecule metabolic process (G0:0043170) & 6824 & 23 & $1.158 \mathrm{e}+01$ & + & $6.303 \mathrm{e}-03$ \\
\hline \multicolumn{6}{|c|}{ (a) Part of GO enrichment results of up-regulated DEGs } \\
\hline Term & $\begin{array}{l}\text { Background } \\
\text { frequency }\end{array}$ & $\begin{array}{l}\text { Sample } \\
\text { frequency }\end{array}$ & Expected & $+\%$ & P-value \\
\hline molecular_function (GO:0003674) & 15480 & 296 & $2.336 e+02$ & + & $8.952 e-15$ \\
\hline transmembrane transporter activity (GO:0022857) & 934 & 44 & $1.409 \mathrm{e}+01$ & + & $5.370 \mathrm{e}-09$ \\
\hline substrate-specific transmembrane transporter activity (GO:0022891) & 852 & 41 & $1.286 e+01$ & + & $1.431 \mathrm{e}-08$ \\
\hline transporter activity ( $\underline{\underline{G 0}: 0005215)}$ & 1204 & 48 & $1.817 \mathrm{e}+01$ & + & $1.623 \mathrm{e}-07$ \\
\hline substrate-specific transporter activity (GO:0022892) & 985 & 42 & $1.486 e+01$ & + & $2.987 \mathrm{e}-07$ \\
\hline active transmembrane transporter activity (GO:0022804) & 305 & 22 & $4.602 e+00$ & + & 4.134e-07 \\
\hline cation transmembrane transporter activity (GO:0008324) & 602 & 31 & $9.084 \mathrm{e}+00$ & + & $7.033 \mathrm{e}-07$ \\
\hline secondary active transmembrane transporter activity (G0:0015291) & 190 & 17 & $2.867 e+00$ & + & $1.286 \mathrm{e}-06$ \\
\hline metal ion transmembrane transporter activity (G0:0046873) & 394 & 24 & $5.945 \mathrm{e}+00$ & + & $1.946 \mathrm{e}-06$ \\
\hline ion transmembrane transporter activity (G0:0015075) & 785 & 35 & $1.184 \mathrm{e}+01$ & + & $2.516 \mathrm{e}-06$ \\
\hline receptor activity (GO:0004872) & 1493 & 52 & $2.253 e+01$ & + & $2.761 \mathrm{e}-06$ \\
\hline signal transducer activity (GO:0004871) & 1569 & 53 & $2.367 e+01$ & + & $5.339 \mathrm{e}-06$ \\
\hline molecular transducer activity (GO:0060089) & 1569 & 53 & $2.367 \mathrm{e}+01$ & + & $5.339 \mathrm{e}-06$ \\
\hline
\end{tabular}


compound metabolic process (GO:0006725) (20 genes) and regulation of metabolic process (GO:0019222). And the down-regulated DEGs mostly involve ion binding (GO:0043167) (158 genes),multicellular organismal process(GO:0032501)(127 genes), single-multicellular organisms process(GO:0044707) (121 genes) and response to stimulus (GO:0050896) (158 genes) etc.

We used a online tool GATHER [20] to do pathway enrichment (Figure 3). The up-regulated DEGs mostly involve the pathways related to "metabolism". However, the down-regulated DEGs are included in toll-like receptor signaling pathway, gamma-hexachlorocyclohexane degradation besides two metabolism-associated pathways.

We also investigated the clustering of the DEGs associated with the bladder cancer. The heatmap of the DEGs is showed in Figure 4. From the heatmap, we can see the clustering results of the DEGs. The clustering method can correctly divide the samples into two classes.

\section{Construction and analysis of two DCNs}

We first calculated a adjacency matrix of DEGs at the normal or cancer state using the method "Pearson correlation" based on the gene expression values. If the adjacency value of a gene pair is greater than 0.8 , the two genes will be connected to be an edge of a DCN. We constructed a normal DCN and a cancer DCN (Figure 5) employing the package "WGCNA". We can easily see that in the cancer state, a few up-regulated DEGs take parts in the co-expression relations. Through the comparisons of two different DCNs, we found that the shortest path length distribution (Additional file 3) has a little changes from the normal to the cancer. However, the average clustering coefficient distribution (Figure 6) and the topological coefficients (Additional file 4) under the normal condition differ from those under the cancer conditions, which indicates that the modules in the two different DCNs have a lot of changes.

Next, we want to find differential links (edges) between two different DCNs. At first, we set two thresholds T1 and T2. If the correlation (Here is pearson correlation) of a gene pair is less than T1 (Here is set 0.3) at normal state, but is bigger than T2 (Here is set 0.8) at the cancer state, the link of the gene pair is defined as a differential link. We computed the significance of the differential links using permuation test (p-value $<2.2 \mathrm{e}$ 16). All the selected differential links can compose a
KEGG Pathway

1.path.hsa00460: Cyanoamino acid metabolism

2. patthissa0240: Pyimidine metabolism

3.path.hssa06080: Methane metabolism

4. path.hsa00271: Methionine metabolism

5. pathinsa00670: One carbon pool by yolate

6. pathihsa00230: Purine metabolism

7. pathinsa00020: Citrate cycle (TCA cycle)
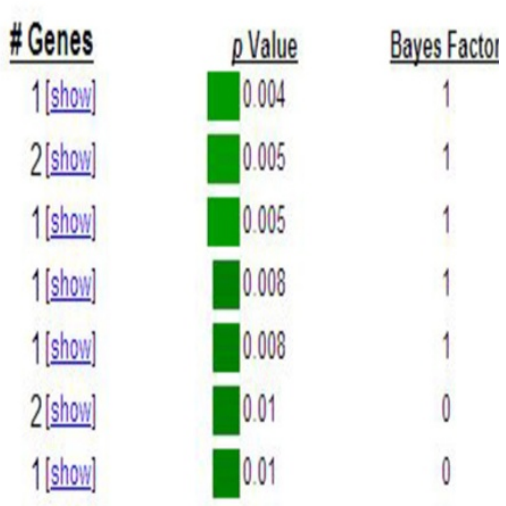

(a) The KEGG enrichment results of up-regulated DEGs
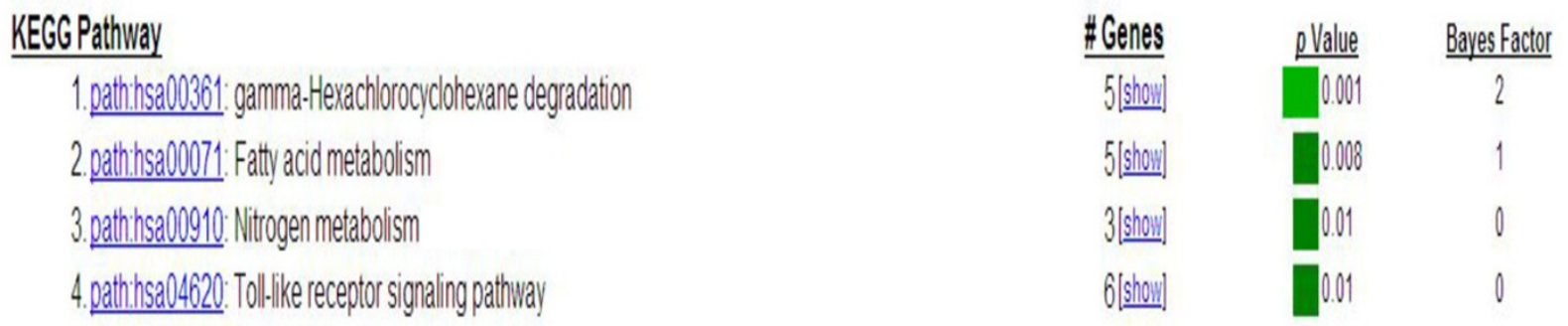

(b)The KEGG enrichment results of down-regulated DEGs

Figure 3 The KEGG enrichment results of DEGs associated with the bladder. 


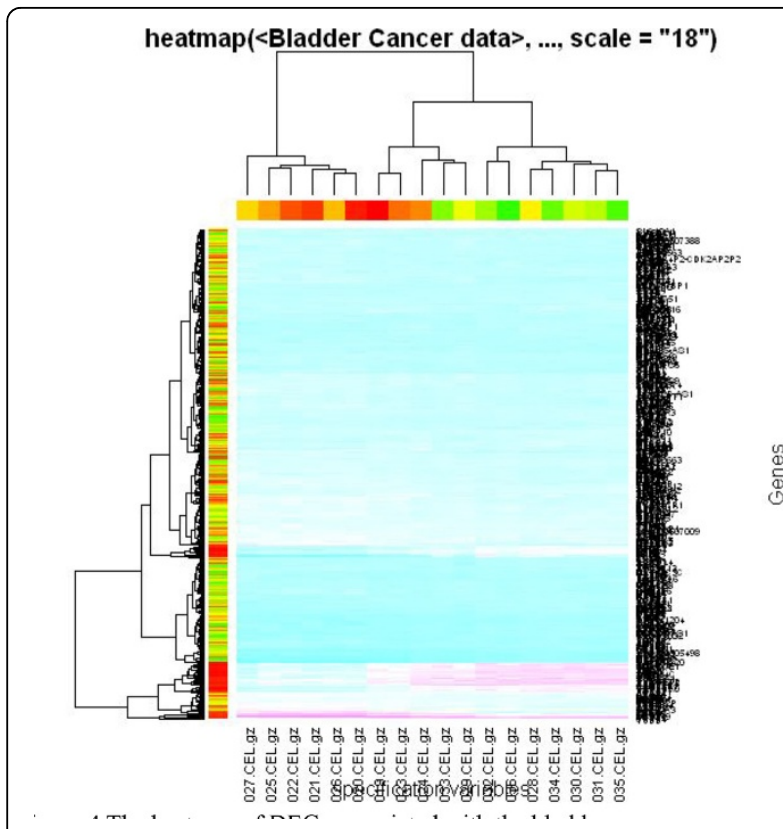

Figure 4 The heatmap of DEGs associated with the bladder cancer.

differential co-expression subnetwork (Figure 7). In Figure 7, the blue ellipses represent down-regulated DEGs and the red ellipses represent up-regulated DEGs. The shapes of nodes (genes) grow bigger with the degree of nodes. The 6 biggest nodes represent the hub genes: "GDF9", “CYP1A2", "ATF7", “TRPM3", “CER1", "PTPRJ", "KCNIP1", and "LRRC15". These hub genes mainly involve the biological processes: cellular response to stimulus (GO:0051716)(5 genes), regulation of biological process (GO:0050789)(7 genes), response to stimulus (GO:0050896)(6 genes), multi-organism process (GO:0051704)(4 genes), and regulation of localization (GO:0032879)(4 genes). And they mostly take part in five pathways: gamma-Hexachlorocyclohexane degradation (path:hsa00361), Fatty acid metabolism((path: hsa00361)), Adherens junction(path:hsa04520), Tryptophan metabolism(path:hsa00380), and Wnt signaling pathway(path:hsa04310). Among of them, "CYP1A2" and "PTPRJ" have been reported that they are associated with the bladder cancer [21-24].

And then, we dectected the modules of a DCN. There have been some methods using clustering algorithms [25-32]. Here, we adopted another good approach [33]. In order to detect the modules of two DCNs under different conditions, we begun with calculating the topological overlap matrix (TOM) [33] of expression datasets. The topological overlap of two nodes reflects their similarity in terms of the commonality of the nodes they connect to. In order test and verify the inference, we clustered the TOM similarities

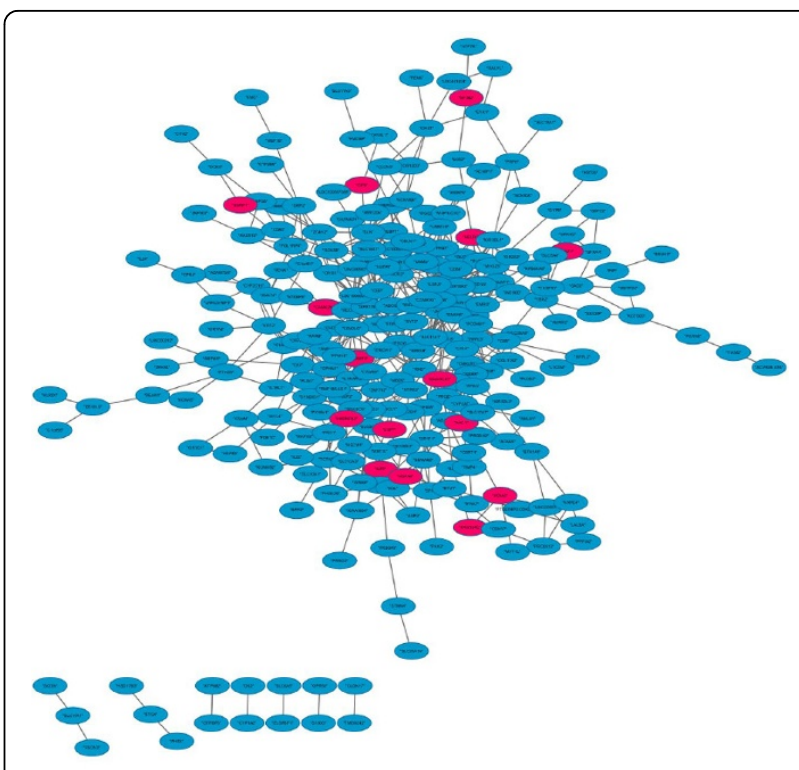

(a) The DCN at normal state

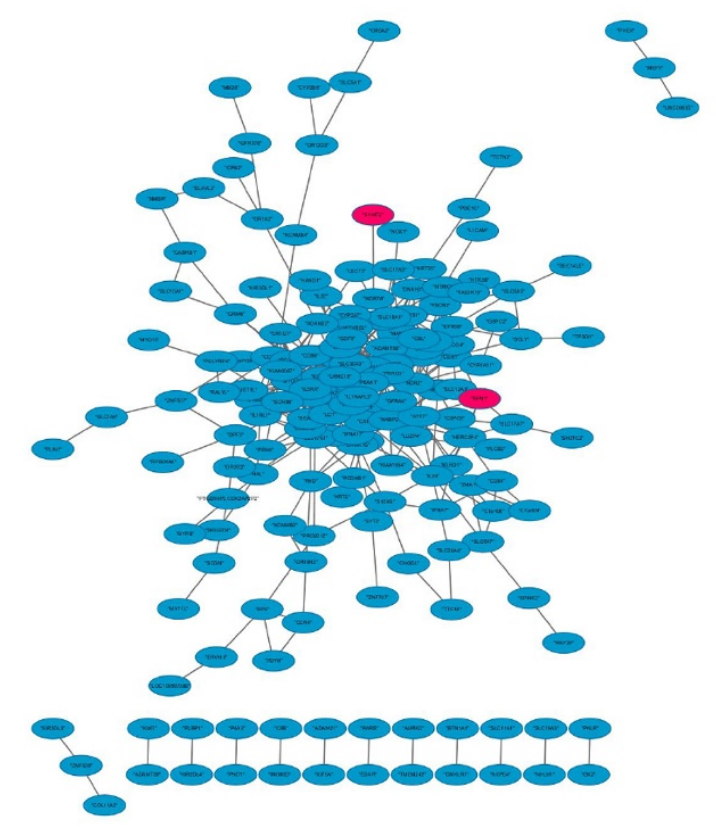

(b) The DCN at cancer state

Figure 5 Two DCNs at normal and cancer state cancer. The blue ellipses represent down-regulated DEGs and the red ellipses represent up-regulated DEGs. Comparing with the normal DCN, in the cancer DCN, the up-regulated DEGs decrease. In additional, the structure of two differential DCNs change a lot.

from two different conditions (Additional file 5). From the Additonal file 5, it is obvious that the modules at two different states change greatly. For observing the module changes, we plotted the module heatmaps of 


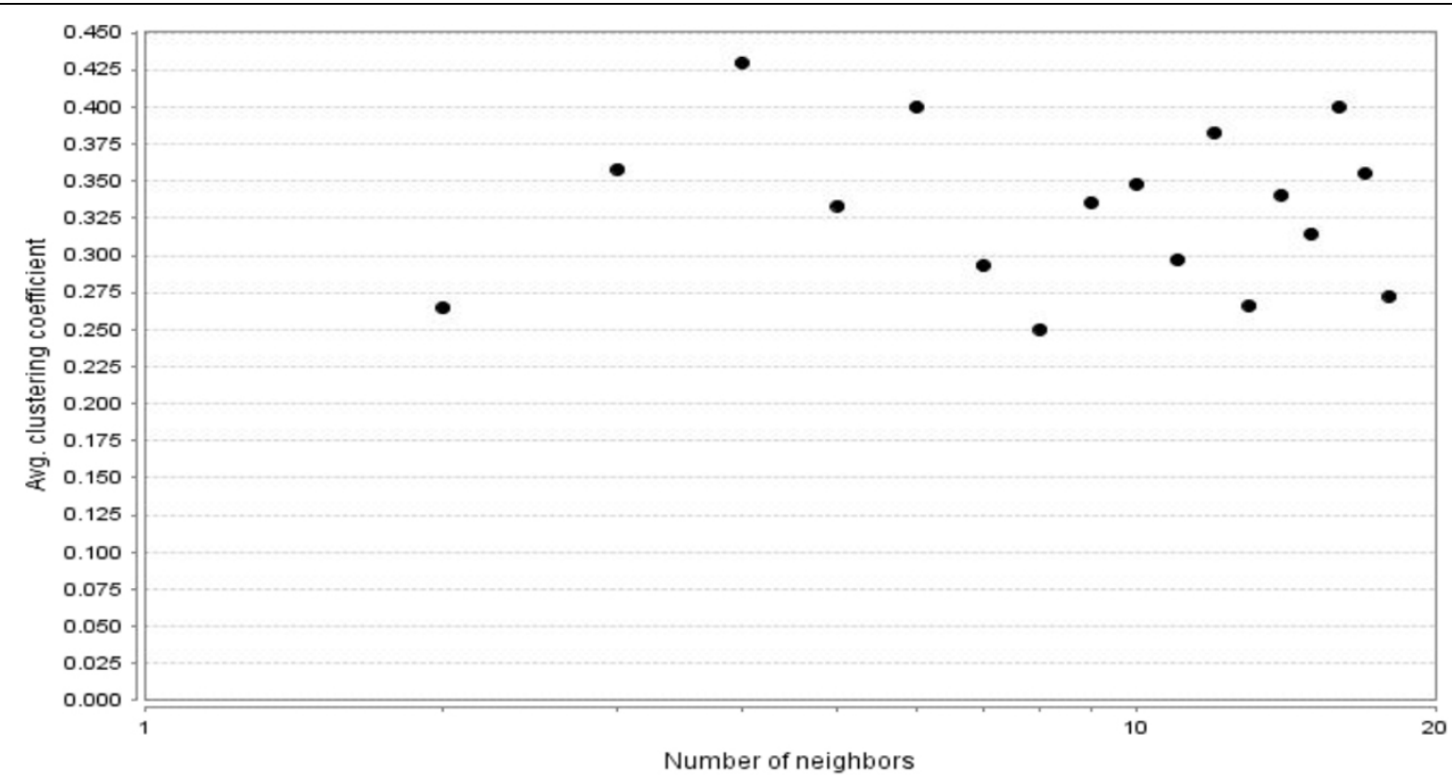

(a) The normal state

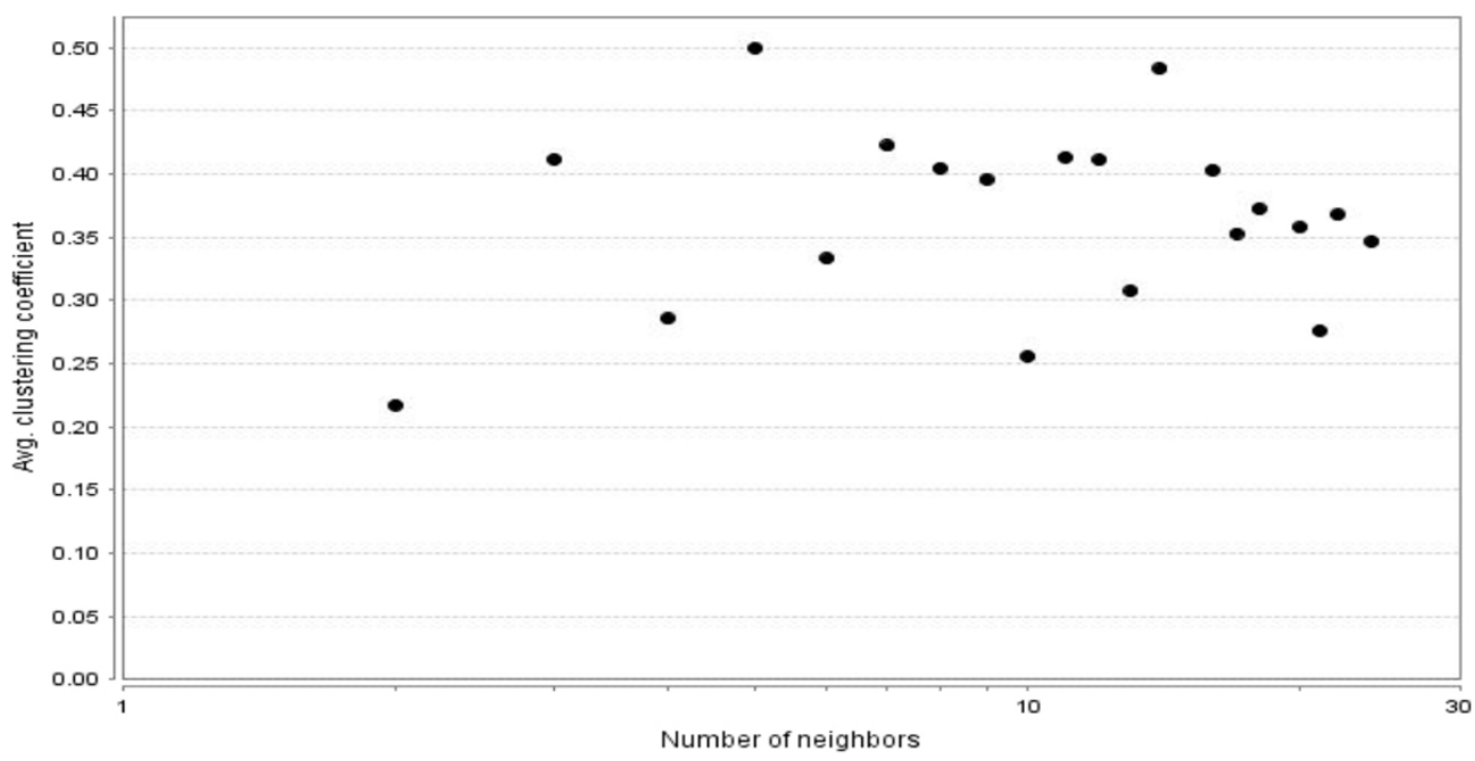

(b) The cancer state

Figure 6 The avg. clustering coefficient distribution of two different DCNs. For the normal DCN, the average clustering coefficient distribution is bigger, implying that the cancer DCN has more modules than the normal DCN.

DEGs at two different states and showed parts of the module heatmaps (Figure 8).

\section{Discussion}

The development of molecular markers for tumor classification and expression signatures that predict outcome will greatly improve diagnosis treatment of bladder cancer. We employed the R package "SAMR" to select 392 DEGs including 36 up-regulated and 356 downregulated. In the GO function enrichment results (Additional file 6 Additional file 7), it is showed that the main functions of the DEGs are cellular physiological precess (24 up-regulated DEGs; 167 down-regulated DEGs) and cellular metabolism (19 up-regulated DEGs; 104 down- 


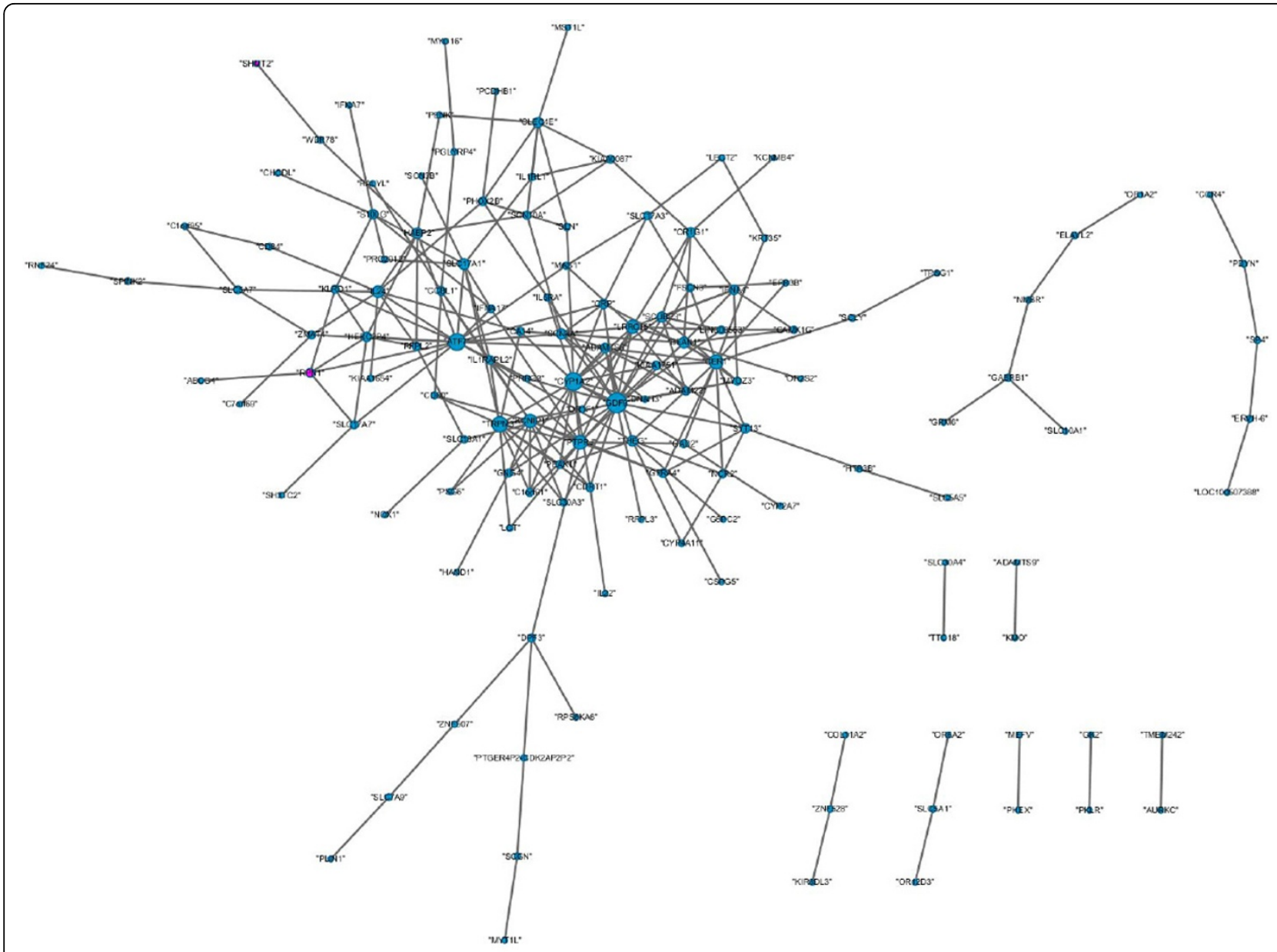

Figure 7 Differential co-expression subnetwork of two different DCNs. The blue ellipses represent down-regulated DEGs and the red ellipses represent up-regulated DEGs. The shapes of nodes (genes) grow bigger with the degree of nodes. The 6 biggest nodes represent the hub genes in the subnetwork.

regulated DEGs), which is reasonable for the bladder. However, it is not clear why the difference between the number of the up-regulated and the down-regulated is so large.

We used the R packages "WGCNA" to construct two DCNs under different conditions. And we used the tool "Cytoscape" to visualize and analyze the two different DCNs. Some hub genes were found and analyzed in view of bioinformatics. The hub genes of the normal DCN mainly involve the neuroactive ligand-receptor interaction pathway and their GO functions mostly are response to biotic stimulus, response to stimulus and response to external stimulus. The hub genes of the cancer DCN involve the following three KEGG pathways: gamma-Hexachlorocyclohexane degradation, Fatty acid metabolism and Tryptophan metabolism. Their main GO functions are cellular physiological process, surface receptor linked signal transport and signal transduction. In addition, we found some difference of the two DCNs in modules from the clustering plots and the heatmaps. But it is unknown for us that the functions of the different modules, which is our future work.

We found several hub genes from the selected differential co-expression subnetwork of two different DCNs. Two of them have been reported to be associated with the bladder cancer. Then whether are the other hub genes associated with the bladder cancer? It need to be validated through biological experiments.

\section{Conclusions}

In the work, we adopted an integrated strategy to analyzing the bladder cancer-associated genes by combining several $R$ packages, Gene Ontology and KEGG. In the experimental results, it shows that the bladder cancer results from the abnormal signaling pathways caused by many genes. Through the data mining for gene expression microarrays, we found differential co-expression subnetwork and the hub genes of the subnetwork. 


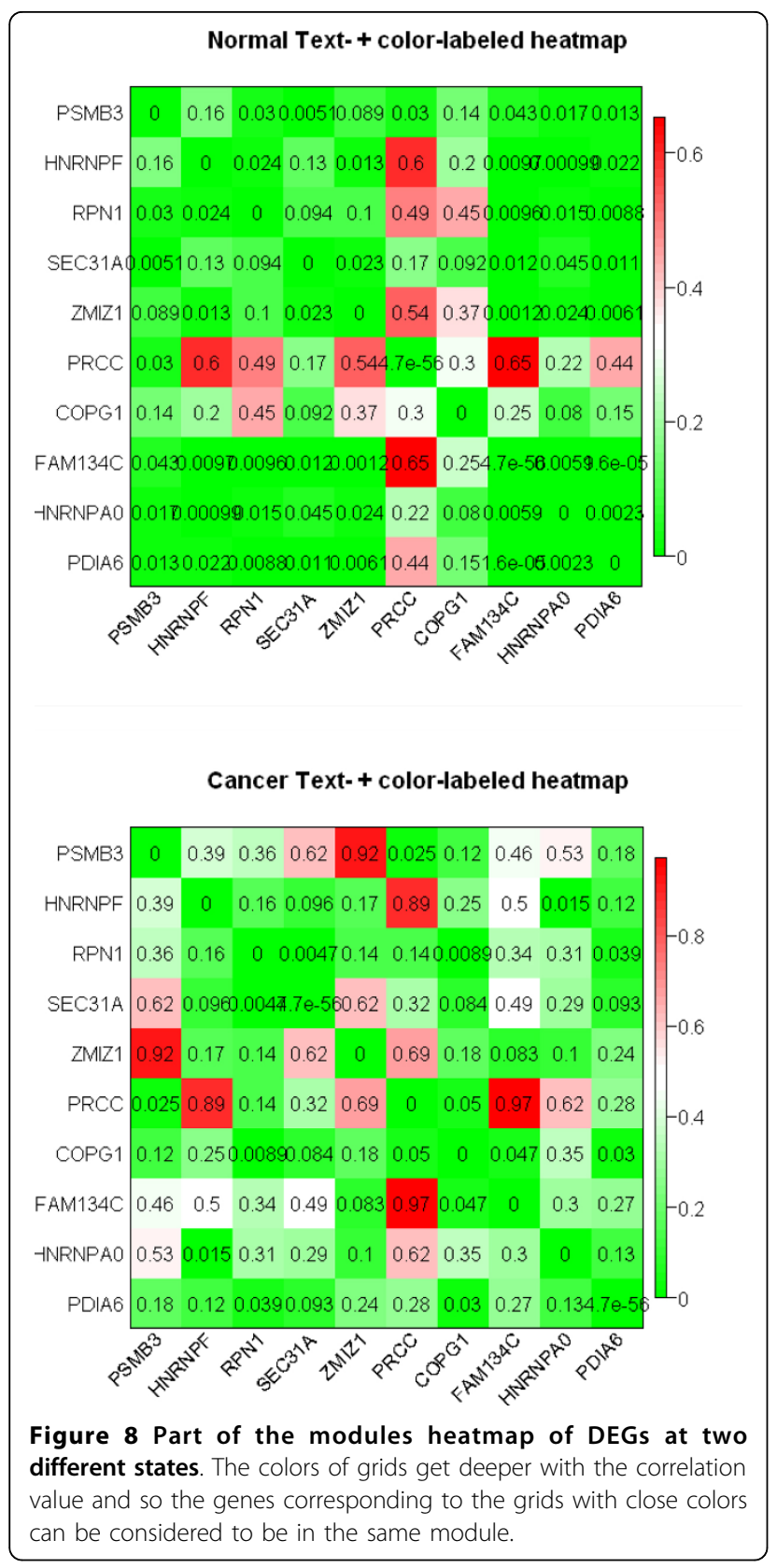

Through the main GO functions and pathways of the hub genes, we can better understand the development of the bladder cancer, which will support the wet biological experiment and even further promote the prevention, treatment,diagnosis and cure of the bladder cancer in the future.

\section{Methods}

\section{Selecting differential expressed genes}

We adopted the method called "Significance analysis of microarrays (SAM)" [15] to pick out the DEGs. The selection approach is based on analysis of random fluctuations in the data. To account for gene-specific fluctuations, they defined a statistic based on the ratio of change in gene expression to standard deviation in the data for that gene. The "relative difference" $d(i)$ in gene expression is:

$$
d(i)=\frac{\bar{x}_{I}(i)-\bar{x}_{U}(i)}{s(i)+s_{0}}
$$

Where $\bar{x}_{I}(i)$ and $\bar{x}_{U}(i)$ are defined as the average levels of expression for gene $(i)$ in states $\mathrm{I}$ and $\mathrm{U}$, respectively. The "gene-specific scatter" $s(i)$ is the standard deviation of repeated expression measurements:

$$
s(i)=\sqrt{a\left\{\sum_{m}\left[x_{m}(i)-\bar{x}_{I}(i)\right]^{2}+\sum_{n}\left[x_{n}(i)-\bar{x}_{U}(i)\right]^{2}\right\}}
$$

Where $\sum_{m}$ and $\sum_{n}$ are summations of the expression measurements in states I and U, respectively, $a=\left(1 / n_{1}+\right.$ $\left.1 / n_{2}\right) /\left(n_{1}+n_{2}-2\right)$, and $n_{1}$ and $n_{2}$ are the numbers of measurements in states I and U.

To find significant change in gene expression, genes were ranked by magnitude of their $d(i)$ values, so that $d(i)$ was the $i$ th largest relative difference. For each of the $N$ balanced permutations relative differences $d_{p}(i)$ were also calculated, and the genes were again ranked such that $d_{p}(i)$ was the $i$ th largest relative difference for permutation $p$. The expected relative difference, $d_{E}(i)$, was defined as the average over the $N$ balanced permutations, $d_{E}(i)=\sum_{p} d_{p}(i) / N$.

To identify potentially significant changes in expression, they used a scatter plot of the observed relative difference $d(i)$ vs. the expected relative difference $d_{E}(i)$. For the vast majority of genes, $d(i) \cong d_{E}(i)$, but some genes are represented by points displaced from the $d(i)=d_{E}(i)$ line by a distance greater than a threshold $\Delta$ and these genes were called "significant genes".

The method for setting thresholds provides asymmetric cutoffs for induced and repressed genes. The alternative is the standard $t$ test, which improves a symmetric horizontal cutoff, with $d(i)>c$ for induced genes and $d(i)<c$ for repressed genes.

\section{Detecting modules in differential co-expression networks}

We first needed to construct topological overlap matrices (TOM) [33,34]. The topological overlap is for measuring pair-wise similarity. They start with a network encoded by its corresponding adjacency matrix $A=\left[a_{i j}\right]$ which is a symmetric with binary entries. By convention, the diagonal elements are assumed to be zero.

The topological overlap of two nodes reflects their similarity in terms of the commonality of the nodes they connect to. Ravasz et al. [35] define the topological overlap matrix $T=\left[t_{i j}\right]$ as follows 


$$
t_{i j}= \begin{cases}\frac{l_{i j}+a_{i j}}{\min \left\{k_{i}, k_{j}\right\}+1-a_{i j}} & \text { if } i \neq j \\ 1 & \text { if } i=j\end{cases}
$$

Where, $l_{i j}=\sum_{u} a_{i u} a_{u j}, k_{i}=\sum_{u} a_{i u}$ and the index $u$ runs across all nodes of the network.

Yip and Horvath [33] generalized the TOM of Ravasz et al. [35] by the observation that formula (1) as follows:

$$
t_{i j}= \begin{cases}\frac{\left|N_{1}(i) \cap N_{1}(j)\right|+a_{i j}}{\min \left\{\left|N_{1}(i)\right|,\left|N_{1}(j)\right|\right\}+1-a_{i j}} & \text { if } i \neq j \\ 1 & \text { if } i=j\end{cases}
$$

Where $N_{1}(i)$ denotes the set of neighbors of $I$ excluding $I$ itself and $|\cdot|$ denotes the number of elements (cardinality) in its argument. The quantity $\left|N_{1}(i) \cap N_{1}(j)\right|$ measures the number of common neighbors that nodes $i$ and $j$ shares whereas $\left|N_{1}(i)\right|$ gives the number of neighbors of $i$.

By denoting $N_{m}(i)$ (with $m>0$ ) the set of nodes (excluding $i$ itself) that are reachable from $i$ within a path of length $m$, i.e.,

$$
N_{m}(i):=\{j \neq i \mid \operatorname{dist}(i, j) \leq m\}
$$

Where $\operatorname{dist}(i, j)$ is the geodesic distance between $i$ and $j$, then a very natural generalization of the TOM can be read as follows

$$
t_{i j}^{[m]}= \begin{cases}\frac{\left|N_{m}(i) \cap N_{m}(j)\right|+a_{i j}}{\min \left\{\left|N_{m}(i)\right|,\left|N_{m}(j)\right|\right\}+1-a_{i j}} & \text { if } i \neq j \\ 1 & \text { if } i=j\end{cases}
$$

The matrix $T^{[m]}=\left[t_{i j}^{[m]}\right]$ is called the $m$ - th order generalized topological overlap matrix (GTOMm). This quantity simply measures the agreement between the nodes that are reachable from $i$ and from $j$ within $m$ steps.

\section{Additional material}

\section{Additional file 1:}

Additional file 2:

Additional file 3:

Additional file 4:

Additional file 5:

Additional file 6 :

Additional file 7 :

\section{Competing interests}

The authors declare that they have no competing interests.

\section{Authors' contributions}

Su-Ping Deng mainly designed all the experiment, did the implementation of the design and wrote all the manuscript. Lin Zhu collected the experimental datasets and preprocessed the datasets. De-Shuang Huang was responsible for the supervision and direction of all the work. Zhu-Hong You revised the manuscript and programmed a part of $R$ resource.

\section{Acknowledgements}

This work was supported by the grants of the National Science Foundation of China(61133010, 61303111, 61373105,30711140358, 61472280 and 6140050774), the National High-Tec h R\&D Program (863) (2014AA021502).

\section{Declarations}

The publication costs for this article were partly funded by the grants of the National Science Foundation of China(61133010, 61303111, 61373105, $30711140358,61472280$ and 6140050774$)$ and partly supported by the National High-Tec h R\&D Program (863) (2014AA021502). And Publication costs for this article were also funded by the corresponding author's institution.

This article has been published as part of BMC Genomics Volume 16 Supplement 3, 2015: Selected articles from the 10th International Symposium on Bioinformatics Research and Applications (ISBRA-14): Genomics. The full contents of the supplement are available online at http:// www.biomedcentral.com/bmcgenomics/supplements/16/S3.

Published: 29 January 2015

\section{References}

1. Tosini I, Wagner U, Sauter G, Egloff M, Knogagil H, Alund G, Bannwart F, Mihatshg MJ, Gasser TC, Maurer R: Clinical significance of interobserver differences in the staging and grading of superficial bladder cancer. BJU Int 2000, 85:48-53.

2. Oosterlinck W, Lobel B, Jakse G, Malmstrom P-U, Stockle M, Sternberg C Guidelines on bladder cancer. European Urology 2002, 41:105-112.

3. Wang B, Huang DS, Jiang C: A new strategy for protein interface identification using manifold learning method. IEEE Transactions on NanoBioscience 2014, 13(2):118-123.

4. Huang DS, Zhang L, Han K, Deng S, Yang K, Zhang H: Prediction of protein-protein interactions based on protein-protein correlation using least squares regression. Current Protein \& Peptide Science 2014, 15(6):553-560.

5. Xia J-F, Zhao X-M, Song J, Huang DS: APIS: accurate prediction of hot spots in protein interfaces by combining protrusion index with solvent accessibility. BMC Bioinformatics 2010, 11:174

6. Stuart $\mathrm{JM}$, et al: A gene-coexpression network for global discovery of conserved genetic modules. Science 2003, 302:249-255.

7. Bergmann $\mathrm{S}$, et al: Similarities and differences in genome-wide expression data of six organisms. PLOS Biol 2004, 2:E9.

8. Lee HK, et al: Coexpression analysis of human genes across many microarray data sets. Genome Res 2004, 14:1085-1094.

9. Noort W, et al: The yeast coexpression network has a small-world, scalefree architecture and can be explained by a simple model. EMBO rep 2004, 5:280-284

10. Hudson NJ, Reverter A, Dalrymple BP: A differential wiring analysis of expression data correctly identifies the gene containing the causal mutation. PLoS Comput Biol 2009, 5:e1000382.

11. Reverter A, Hudson NJ, Nagaraj SH, Perez-Enciso M, Dalrymple BP: Regulatory impact factors: unraveling the transcriptional regulation of complex traits from expression data. Bioinformatics 2010, 26:896-904

12. Staehler CF, Keller A, Backes C, Chandran A, et al: Whole miRNome-wide differential co-expression of microRNAs. Genomics Proteomics Bioinformatics 2012, 10:285-294.

13. Bhattacharyya M, Bandyopadhyay S: Studying the Differential Coexpression of MicroRNAs Reveals Significant Role of White Matter in Early Alzheimer's Progression. Molecular BioSystems 2013, 9:457-466.

14. Smyth GK: Limma: linear models for microarray data. Bioinformatics and Computational Biology Solutions using $R$ and Bioconductor, $R$ Springer New York; 2005, 397-420.

15. Tusher VG, Tibshirani R, Chu G: Significance analysis of microarrays applied to the ionizing radiation response. PNAS 2001, 98:5116-5121. 
16. Langfelder P, Horvath S: WGCNA: an R package for weighted correlation network analysis. BMC Bioinformatics 2008, 9:559.

17. Cytoscape. [http://www.cytoscape.org/].

18. GEO. [http://www.ncbi.n/m.nih.gov/geo/].

19. AmiGO. [http://amigo.geneontology.org/amigo].

20. GATHER. [http://gather.genome.duke.edu/].

21. Altayli E, Gunes S, Yilmaz AF, Goktas S, Bek Y: CYP1A2, CYP2D6, GSTM1, GSTP1, and GSTT1 gene polymorphisms in patients with bladdercancer in a Turkish population. Int Urol Nephrol 2009, 41(2):259-66.

22. Pavanello S, Mastrangelo G, Placidi D, Campagna M, Pulliero A, Carta A, Arici C, Porru S: CYP1A2 polymorphisms, occupational and environmental exposures and risk of bladder cancer. Eur J Epidemiol 2010, 25(7):491-500.

23. Obara W1, Ohsawa R, Kanehira M, Takata R, Tsunoda T, Yoshida K, Takeda K, Katagiri T, Nakamura Y, Fujioka T.: Cancer peptide vaccine therapy developed from oncoantigens identified through genome-wide expression profile analysis for bladder cancer. Jpn J Clin Oncol 2012 42(7):591-600, doi: 10.1093/jjco/hys069. Epub 2012 May 25.

24. Wang L, Matoso A, Sciandra KT, Yakirevich E, Sabo E, Zhang Y, Meitner PA, Tavares R, Noble L, Pareek G, DeLellis RA, Resnick MB: Expression of S100A4 in renal epithelial neoplasms. Appl Immunohistochem Mol Morphol 2012, 20(1):71-6, doi: 10.1097/PAl.0b013e31821fc8b7.

25. Wang S-L, Zhu Y, Jia W, Huang DS: Robust classification method of tumor subtype by using correlation filters. IEEE/ACM Transactions on Computational Biology and Bioinformatics 2012, 9(2):580-591.

26. Zheng C-H, Zhang L, To-Yee Ng V, Shiu SC, Huang DS: Molecular pattern discovery based on penalized matrix decomposition. IEEE/ACM Transactions on Computational Biology and Bioinformatics 2011, 8(6):1592-1603.

27. Zheng C-H, Zhang L, To-Yee Ng V, Shiu SC, Huang DS: Metasample-based sparse representation for tumor classification. IEEE/ACM Transactions on Computational Biology and Bioinformatics 2011, 8(5):1273-1282.

28. Zheng C-H, Huang DS, Zhang L, Kong X-Z: Tumor clustering using nonnegative matrix factorization with gene selection. IEEE Transactions on Information Technology in Biomedicine 2009, 13(4):599-607.

29. Liu K-H, Huang DS: Cancer classification using rotation forest. Computers in Biology and Medicine 2008, 38(5):601-610.

30. Shun P, Huang DS: Cooperative competition clustering for gene selection. Journal of Cluster Science 2006, 17(4):637-651.

31. Zheng $\mathrm{C}-\mathrm{H}$, Huang DS, Shang L: Feature selection in independent component subspace for microarray data classification. Neurocomputing 2006, 69(16-18):2407-2410.

32. Huang DS, Zheng C-H: Independent component analysis based penalized discriminant method for tumor classification using gene expression data. Bioinformatics 2006, 22(15):1855-1862.

33. Yip AM, Horvath S: The generalized Topological Overlap Matix for Detecting Modules in Gene Networks. Proceedings of the 2006 international conference on bioinformatics \& computational biology, BIOCOMP'06, Las Vegas, Nevada, USA, June 26-29 2006

34. Zhang B, Horvath S: A General Framework for Weighted Gene CoExpression Network Analysis. Statistical Applications in Genetics and Molecular Biology 2005, 4(1):Article 17.

35. Ravasz E, Somera A, Mongru D, Oltvai Z, Barabasi A: Hierarchical organization of modularity in metabolic networks. Science 2002 297(5586):1551-1555.

\section{Submit your next manuscript to BioMed Central and take full advantage of:}

- Convenient online submission

- Thorough peer review

- No space constraints or color figure charges

- Immediate publication on acceptance

- Inclusion in PubMed, CAS, Scopus and Google Scholar

- Research which is freely available for redistribution

Submit your manuscript at www.biomedcentral.com/submit 